\title{
Using Handwriting Evaluation Software to Predict and Increase Diagnosis for Parkinson's
}

\author{
Jordan King ${ }^{1}$ and Soo Park ${ }^{1}$ \\ ${ }^{1}$ Advanced Technologies Academy, Las Vegas, NV, USA
}

\begin{abstract}
Around the world, there currently exists a problem when it comes to the diagnosis of Parkinson's disease (PD). Unfortunately, nearly half of all Americans who have PD remain undiagnosed, which is problematic when one considers the implications of such ignorance. People who continue to be undiagnosed do not have access to special treatments, therapies, and medications that would help alleviate the symptoms of PD and decrease the burden of it altogether. Fortunately, amidst recent technological advancements in computing and the contemporary paradigm shift to using handwriting as a diagnosis method for PD, a shimmer of hope reveals itself. By using a machine learning software program that predicts a user's likelihood of having PD through their handwriting alone, people might feel more inclined to seek a formal evaluation for the disease. Since it is rather inexpensive, based on concrete, quantitative kinematics of an individual's handwriting, and holds legitimacy due to the existence of similar evaluation programs, the software could help increase the amount of people that seek a formal PD evaluation and diagnosis.
\end{abstract}

\section{Introduction}

Ever since James Parkinson's publication of “An Essay on the Shaking Palsy" in 1817, common criteria and methods for the diagnosis of Parkinson's disease (PD) have been employed by neurologists around the globe. Over 200 years later, these methods have, for the most part, stayed consistent, with symptoms such as resting tremor and fine motor control complications remaining front and center (Parkinson, 1817). However, with the introduction of new technologies and PD research Parkinson could have only dreamed of, the handwriting analyzation method of diagnosis has become commonplace. Additionally, the contemporary advancements of computer science and algorithmic computation have provided new ways to standardize and automate the diagnosis process. One of such innovations is a new proposal: a computer software program that predicts an individual's likelihood of having PD based on their handwriting. By inscribing a series of geometric figures and letter characters onto a drawing tablet, this program could theoretically be capable of diagnosing PD in an individual by assessing the characteristics of their handwriting, such as the velocity, pressure, and elapsed time it took to write the prescribed tasks.

However, amidst these newfound clinical tests for PD, one primary issue still exists: around 40 percent of people with PD in the United States alone may not be formally diagnosed with it (Lan \& Shih, 2014). While this might be due in part by the cost of healthcare in the country, this notion is immensely dangerous because by avoiding or missing a formal clinical diagnosis of PD, an individual's prospects for life become increasingly diminished. According to research conducted by Walker et al., "Physiotherapists, occupational therapists, dietitians, speech and language therapists and nurse specialists" (2014), all become available upon receiving a formal diagnosis of PD. Thus, without that early diagnosis, patients will fail to receive vital treatment options that would otherwise decrease the debilitating nature of the disease.

For this reason, this research study considers the recent advent in PD diagnosis methods and analyzes whether or not the use of a handwriting evaluation software would aid in alleviating the disastrous underdiagnosis problem. Although formal methods for the diagnosis of PD are used widely among neurologists, it can be reasoned that the 
employment of an algorithmic software program that predicts a user's likelihood of having PD through their handwriting alone is a better alternative because it would be inexpensive, ubiquitous, and have a perception of trustworthiness that would be attractive to those who would not seek a medical consultation otherwise. Therefore, this research sequence will examine whether or not this proposed evaluation software would motivate and persuade at-risk people to seek a formal diagnosis for PD.

\section{Literature Review}

\section{Overview of PD and Traditional Diagnosis}

Before diving deeper into the evaluation software, it is important to first develop an understanding of PD as a whole and the ways in which it is currently diagnosed. As it stands, there is no definitive way to diagnose PD, as it is a complex disease that can affect the human body in more ways than one. In light of this, medical researchers at UC San Diego developed the Movement Disorder Society Clinical Diagnostic Criteria for Parkinson's Disease (MDS-PD Criteria), which provides guidelines and procedures for clinical diagnosis of PD that have become commonplace in medical institutions nationwide (Postuma et al., 2015). Despite PD's inherent neurological symptoms, the MDS-PD Criteria primarily focuses on the motor aspects of the disease because these features predominate the majority of PD cases and are what define the disease altogether. According to the guide, the clinical umbrella term for the variety of movement symptoms that one with PD experiences is called "parkinsonism," which encapsulates conditions such as bradykinesia, resting tremor, and rigidity (Postuma et al., 2015). In light of this, parkinsonism must first be identified in a patient in order to formally diagnose them with PD.

With regard to bradykinesia, the basal ganglia structure in the brain is affected, causing an individual's ability to exhibit fine motor control to severely weaken to the point where even simple movements become slow and strenuous (Berardelli et al., 2001). Due to this, patients typically report "difficulty using small tools and fastening buttons" (Nutt \& Wooten, 2005) upon being evaluated for the condition. As for resting tremor, or tremor coactus, is defined as tremors or involuntary, repeated, shaking motions that one experiences when their limbs are relaxed that go away upon voluntary movement (Jankovic et al., 1999). The only other aspect of parkinsonism is rigidity, which is characterized by a sensation of continuous muscular stiffness followed by dull aches, or "increased resistance when stretching a muscle passively," (Berardelli et al., 1983). With an understanding of these three components of parkinsonism, a neurologist or movement disorder specialist may more accurately identify PD in a patient.

Once an individual reports symptom correlating with any of these conditions, the MDS-PD Criteria outlines a multitude of different tests that a neurologist may utilize to determine PD. For bradykinesia, it suggests evaluating the rhythmic toe and finger tapping speeds of a patient over a short interval. If the patient struggles to keep up with the predetermined rhythm, or if they appear to be tapping at an unintentionally laggard rate, a neurologist can conclude that they are experiencing fine motor inhibition due to bradykinesia (Postuma et al., 2015). The other parkinsonism conditions, resting tremor and rigidity, are assessed via less quantitative means. Oftentimes, a patient merely needs to provide anecdotal evidence or testimony about movement troubles they have been experiencing while physically demonstrating their tremor or pain to their neurologist. Other evaluation methods that are not restricted to PD diagnosis are far more involved and complicated, commonly utilizing functional magnetic resonance imaging (fMRI), motor therapy, and pharmaceuticals. Since the primary cause for PD is the gradual depletion of dopamine (De Lau \& Breteler, 2006), fMRI scans - which analyze the blood flow and neuronal connectivity of the brain-can be conducted to indicate the declining presence of the neurotransmitter (d' Souza, 2019). Additionally, in accordance with the MDS-PD Criteria, if continual prescription to a dopaminergic-enhancing medication alleviates a patient's parkinsonism conditions, that in and of itself can serve as evidence of the fact that they had PD all along (Postuma et al., 2015). 


\section{The Handwriting Software Approach to PD Diagnosis}

Given that the majority of PD symptoms reside in the motor capabilities of an individual (Postuma et al., 2015), a new practical method of diagnosis has been adopted over the years by neurologists. Even though the MDS-PD Criteria does not explicitly detail it, the evaluation of a patient's handwriting has become a staple in the diagnosis of PD, specifically with regard to the identification of micrographia. This phenomenon, which causes a patient to write abnormally small letters, exists in PD patients due to bradykinesia and rigidity (Thomas et al., 2017). Thanks to this ability to identify two staple PD symptoms, the handwriting diagnosis method tends to be superior to the other previously stated methods, a fact that is elevated by the kinematics present in a handwriting sample. Through the use of a digitizing drawing tablet—and more accurately the Pointer Events API for Javascript—-the more intrinsic features of a patient's handwriting can be recorded. This includes the velocity in which they moved the pen, the pressure they exerted onto the tablet, the overall time it took them to complete the handwriting task, the angle at which they held the pen, the actual pixel size of the letters they wrote, etc. ("Pointer events," 2020).

By virtue of its intense ability to be scrutinized and its correlation with iconic PD symptoms, researchers in recent years have researched and developed technology based on this API and others in order to further support the handwriting diagnosis method. For example, one research group stationed across Europe proved that standardized handwriting testing can be used to differentiate between the writings of PD patients and healthy patients. In their study, Smits et al. (2014) asked their participants to draw a series of different geometric figures-a circle with a 120 millimeter diameter, a star with four intersecting lines, and an archimedean spiral—and sentences repeating the characters "elelelel" on a digitizing drawing tablet in order to assess bradykinesia and micrographia characteristics. What they discovered was that participants with PD took significantly longer to complete the handwriting tasks and did so with far slower velocities and accelerations when compared to their healthy counterparts (Smits et al., 2014).

Now, while this study provides an excellent foundation for future standardized PD writing tests, it neglects to ask whether or not a person would even feel compelled to take this test in the first place. As a matter of fact, when it came to selecting the participants for their study, Smits et al. only chose the PD patients "who replied positively" to their request to participate in the study (2014). By failing to consider why a person would respond negatively to the proposal of taking the test, the researchers missed out on a chance to identify whether or not a handwriting test would even appeal to the average person. If this revolutionary standardized test is to lay dormant, strictly guarded by a medical professional, it cannot be guaranteed that more people will seek a formal evaluation and diagnosis for PD, thus the underdiagnosis issue will persist.

That is why in the present study it is crucial to analyze whether or not a version of this standardized test would attract more people to seek a diagnosis. By providing it in the form of a free, ubiquitous software application, there is hope that it will help subside the underdiagnosis problem, but its failure can also provide further insight into this epidemic that has plagued the United States and beyond.

\section{Addressing Healthcare and Underdiagnosis}

Currently, there exists a great deal of contention with regard to healthcare in the U.S., namely the cost and administration of it. According to the Organization for Economic Co-operation and Development (OECD), the U.S. paid the highest for healthcare out of 36 other affluent countries in 2017, beating out developed nations like China, Norway, and Germany by a landslide of $\$ 10,209$ per capita ("Health spending"). In terms of PD, these costs can extend to the price of fMRI scans and neurological evaluations themselves. Due to this dramatic expenditure, a reported 54 percent of Americans delayed healthcare in 2017, fearful of the associated out-of-pocket cost (Carter, 2019).

For this reason, the proposed handwriting evaluation software was designed with the interest of alleviating this financial burden by virtue of its relatively unlimited accessibility. Rather than feeling deterred by the costs of PD tests, this software can be entirely free, granted an individual has access to a computer and a drawing tablet. As a matter of fact, it would not be inane to predict that such software could be placed in pharmacies or grocery stores 
likewise to blood pressure kiosks since they both serve a similar purpose: to alert the user of a potential issue with their health. Therefore, the cost of using the software could be entirely free, effectively motivating an individual to seek a formal diagnosis of PD. On the other hand, if a person is unable to safely transport themself to one of these kiosks, a future iteration of the proposed software could be reincarnated into a free iPad, iPhone, or Android application, thereby allowing people to take the test within the comfort of their homes and without another dime spent. This relative ease of accessibility and cost efficiency is a possible factor that would motivate an individual to seek a formal diagnosis.

\section{Prevalence of Similar Software Elsewhere}

Another aspect of the proposed evaluation software that is predicted to entice and motivate its users is its perceived authenticity and reliability. Therefore, it is important to note how the software is similar to and has been founded off of similar medical technologies that already exist. For instance, in their research on machine learning, Toraman et al. managed to create a program that could predict and diagnose colon cancer via blood plasma samples (Toraman et al., 2019). Such a development is crucial because it demonstrates the profound capability of algorithmic processing in the medical world; it proves that if a straightforward algorithm is able to predict a condition as complex as cancer, then the technology could also be employed in evaluating PD. Now, in its current iteration, the proposed evaluation software does not utilize machine learning, but rather a series of algorithms to make its prediction, which are remarkably different. However, in the future, it could be expanded to using machine learning, which would not only make its predictive capabilities more accurate, but also make the software as a whole seem more official.

Another piece of technology that the proposed evaluation software can be compared to is one created by Walker et al. in their research article, in which they created a pen that utilizes machine learning techniques in order to predict whether or not an individual has PD (2014). It assesses similar kinematics of one's handwriting in order to assess the presence of bradykinesia and micrographia, but it does so through the pen itself, not through the tablet or API that they are using (Walker et al., 2014). Nevertheless, this research sequence is incredibly similar to Walker et al.'s, and it served as partial motivation for this study as a whole. However, what distinctly differentiates the current study from Walker et al.'s is that they, similar to Smits et al.'s work, failed to consider the patient's susceptibility to the test and their willingness to even partake in it. Even though they make the argument that the pen could be used "in the patient's home or in a clinic," (Walker et al., 2014) they do not acknowledge whether or not such a device would even motivate its users to utilize it or if it would even motivate them to receive a formal medical diagnosis for PD. As such, the current research sequence builds off of the idea of Walker et al. and examines whether or not such a predictive technology would drive its users to go to the clinic and receive a formal PD evaluation and diagnosis, thereby reducing the underdiagnosis issue.

\section{Expected Limitations and Solutions}

\section{"Sloppy" Handwriting Interference}

When evaluating the reliability and validity of a handwriting program, one may entertain the notion that people with inherently or purposely illegible ("sloppy") handwriting would yield grossly invalid evaluations. Granted, this is a legitimate issue, since at face value the writings of a PD patient with micrographia and a person with just small handwriting can be indistinguishable. Likewise, a person who writes deliberately or with insubstantial speed can display no difference statistically with a PD patient suffering from bradykinesia. These people would potentially have an evaluation that would result in a false positive, in which they are diagnosed with PD even though, in reality, they just have poor handwriting. 
Nevertheless, this point of contention does not discredit the proposed handwriting evaluation software altogether. To factor out the error caused by poor handwriting, take into consideration again the study conducted by Smits et al., which concluded that handwriting could be used as a standardized method for the diagnosis of PD. In their article, the research team listed the median velocities, accelerations, and writing times for ten PD patients and ten healthy controls (2014). By extrapolating low and high acceptable bounds from the PD patient's median values, certain thresholds can be created from which a piece of handwriting can be more accurately evaluated. With these thresholds active within the software's algorithm, a person with poor handwriting would either come just shy of the lower bound or just above the upper bound required for a positive PD diagnosis. This is far more apt than the alternative, which would just be dictating that if a person's handwriting kinematics scored lower than the median values, they would automatically receive a PD diagnosis, which would inevitably produce a false positive. As for how these thresholds would be created, please refer to the "Methods" section.

\section{Psychological Evaluation}

Another conflict with the proposed handwriting evaluation software is that it only judges a person on the pretense that their PD has manifested into physical symptoms despite the fact that many early indicators of PD are more psychological. Such symptoms include depression and even bouts of cognitive troubles, like dementia ("Parkinson's disease," 2018). While this is indeed true of PD, these conditions are far too broad to be solely attributed to the disease, let alone be used in the diagnosis of it. Take, for instance, depression, which the World Health Organization (WHO) has reported to affect "more than 264 million people of all ages" ("Depression," 2020). With such an extensive amount of people with depression, the root cause of it for every individual case is inherently varied, thus the condition cannot be used to definitely diagnose PD. Moreover, in cases of dementia, the WHO again reports that this condition is substantially prevalent, stating that "around 50 million people have dementia" ("Dementia," 2019). Given this many people have dementia unrelated to $\mathrm{PD}$, it is unwise to incorporate it as a focal diagnostic measure for this evaluation software.

\section{Methods}

Given the work of literature provided, it is clear that a noticeable gap exists, and that is whether or not society would actually be receptive to this software and if it would motivate users to seek a formal evaluation and diagnosis for PD. Thus, this study attempted to address this question by performing a quantitative analysis of this very question with the aid of a newly created handwriting evaluation software called PAWDS.

The data collection process revolved around a program which predicts a participant's likelihood of having PD through their handwriting. However, since this type of technology is either still sparse and emerging or locked by patents, I undertook the responsibility of developing a prototype program. It is also important to note that the study was meant to corroborate the experience the participants had with the software and thereby deduce whether or not such a program could increase the number of people who seek a formal evaluation for PD. Thus, after completing the test and receiving their evaluation, the participant was asked to submit an anonymous Google Forms survey that assessed their satisfaction with the program and how strongly it convinced them to receive a formal evaluation.

The primary population evaluated in this design was middle aged (35-64 year olds) and elderly (65 years old and older) people, but adolescents (18 years old and younger) and young adults (19-34) were also considered so as to broaden the perspectives being recorded and to increase diversity in responses (minimum of ten people per age group are required). Additional reasoning for this inclusion is that since this technology is still developmental, there is a possibility that it will not become fully available until they are much older and capable of developing PD, so understanding their perspective on this software can help predict its future success. Participation in the study was entirely anonymous so as to limit any researcher bias. The participants themselves were acquired by randomly selecting 
individuals from the Las Vegas, Nevada area and a select high school therein. Their consent was ensured by having them electronically sign a consent form towards the beginning of Google Forms survey.

\section{PAWDS (Parkinson's Algorithmic Writing Detection Software)}

The program that I developed for this study is titled the "Parkinson's Algorithmic Writing Detection Software," or PAWDS for short. It is a software application that I programmed using JavaScript, HTML5, CSS, and the ElectronJS framework, which provides a fairly versatile, website-like user interface. In addition to software, a Wacom Intuos 4M digitizing drawing tablet was used so the user can complete the handwriting tasks utilizing the hand form they are most accustomed to, which helped limit variability that would come from improper or uncomfortable form. When the user initiates $P A W D S$, they are greeted with a welcome screen that introduces them to the name of the program and a "Get Started" button that navigates them to the three main PD tests. The initial two involve the task of drawing: one encourages the user to draw a circle on the screen ten times without lifting their stylus up from the tablet (See Figure 1), while the second one requires an archimedean spiral to be inscribed five times (See Figure 2). The last test, however, asks the user to write the letters "e" and "l" in cursive four times each on a dotted line presented on the screen (See Figure 3). Once all of these tests have been completed, the user is shown their PD evaluation score (a percentage of PD likelihood up to $100 \%$ ) and a button that ejects them to the Google Forms survey, from which the quantitative data for this study is accumulated.

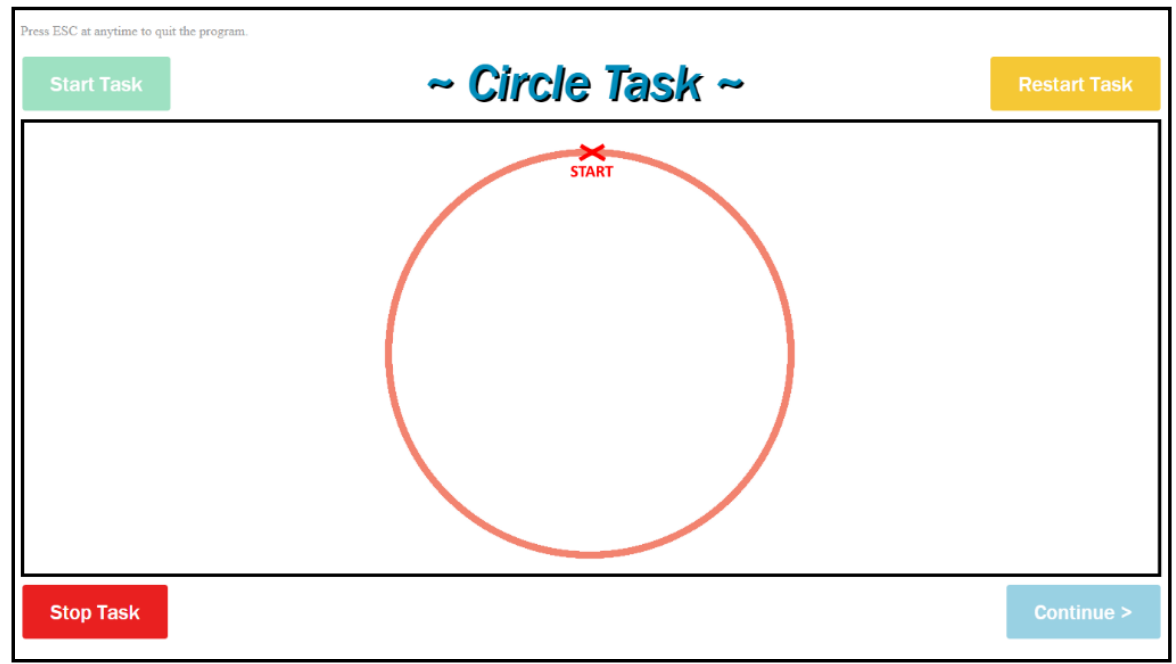

Figure 1. Task where the user must draw a circle 10 times without lifting up their stylus. 


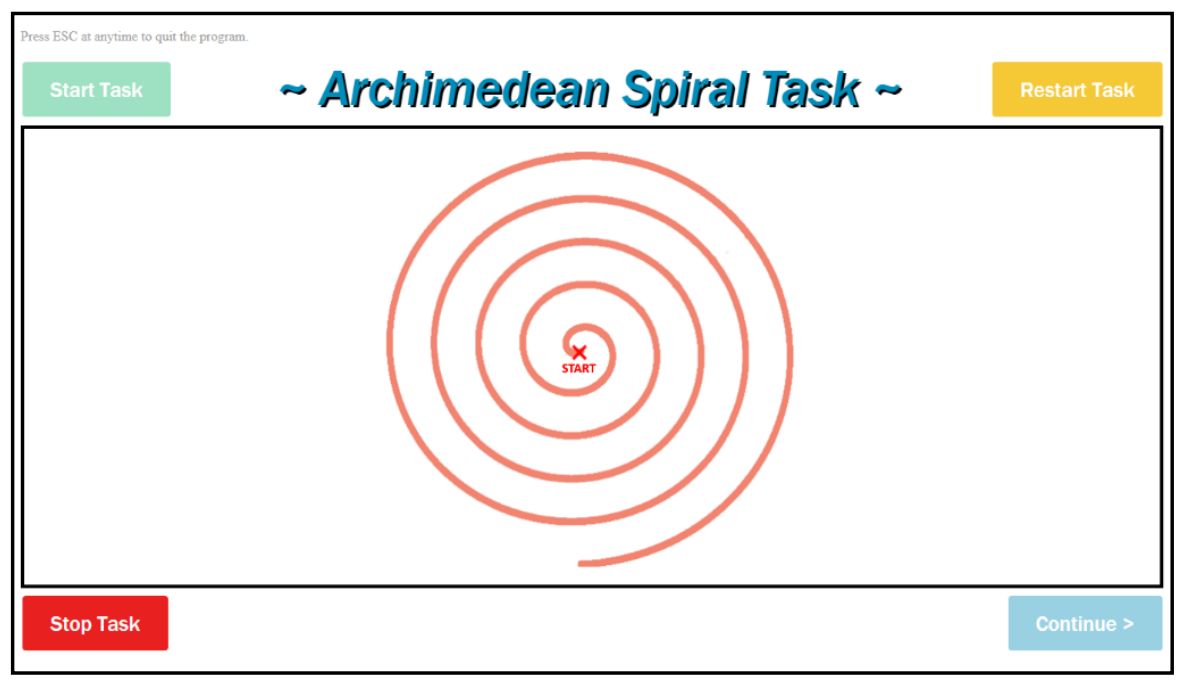

Figure 2. Task where the user must draw an archimedean spiral five times, lifting their stylus up after each successful drawing.

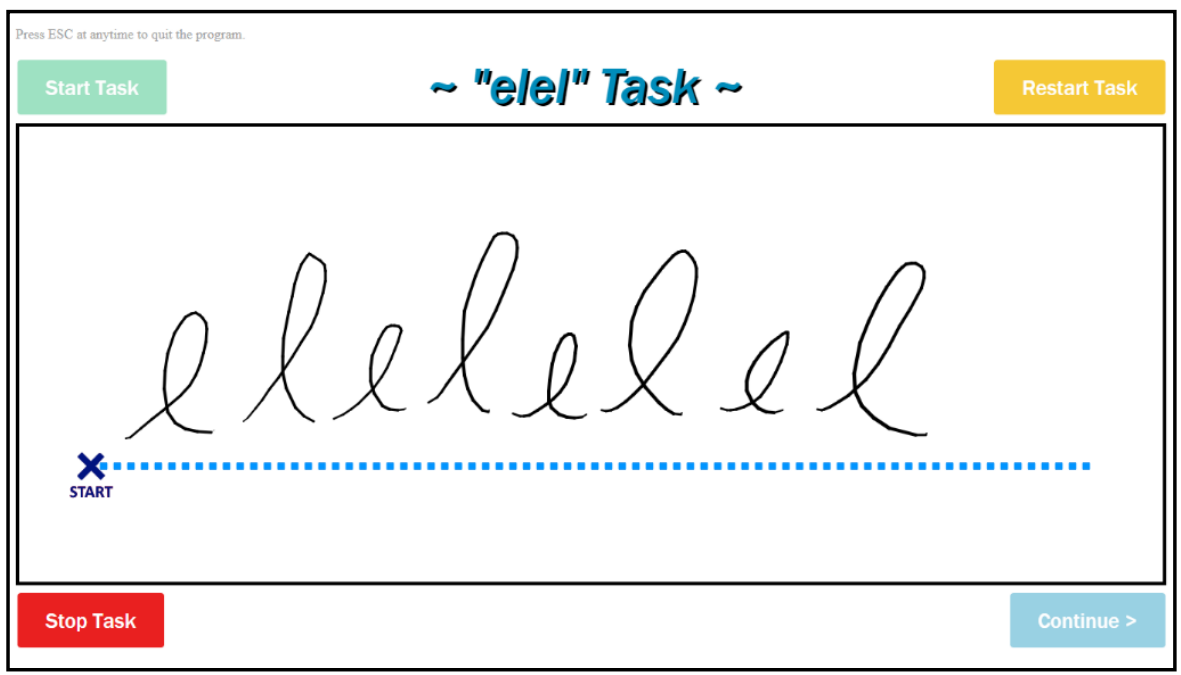

Figure 3. Task where the user must draw the letters "e" and "l" in cursive four times. Here, the letters are written for demonstration purposes, but when the task is actually presented to the user, only the dotted line appears on the canvas.

While it may be true that I am not a professional programmer nor a foremost authority on PD, the development of PAWDS was founded on data collected for the Literature Review. In fact, the tests and the algorithm for the PD evaluation were derived from the methods of neurologist Esther J. Smits et al. 's study on the standardization of handwriting for PD motor symptoms. The tests that were implemented into PAWDS are the very same ones from that study: drawing a circle ten times, inscribing an archimedean spiral five times, and writing "e" and " $\mathrm{l}$ " in cursive four times each. As for how the algorithm evaluates the user, I constructed 90 percent confidence intervals for each quantity that Smits et al. collected, which consisted of the median times it took to each subject to complete each test, the median height of each figure drawn in millimeters, and the median velocities and accelerations of their pen strokes. By using this confidence interval, we can be 90 percent assured that the threshold for each of the handwriting kinematics evaluated for each test will capture a user whose handwriting displays parkisonian characteristics, with the null hypothesis being that the user does not have PD or parkisonian handwriting in general. (As for the intent behind using these intervals, please refer back to the "Sloppy' Handwriting Interference" subsection of the "Expected Limitations and 
Solutions" section.) In practice, this means that if any of my participants' quantities fell within the confidence interval associated with the same test, they would receive a "positive" point, and if they fell above or below the intervals they would receive no points. Once all the tests have been completed, the total number of points obtained is divided by the total number of quantities and attributes tested for. By the time the evaluation screen presents itself to the user, a percentage of how likely they have PD has been calculated and assigned to display in large bold print on the screen.

\section{Participant Satisfaction Survey}

After receiving their PD assessment, the participants were asked to sign a consent form and complete a short Google Forms survey. It is paramount to reiterate that this survey was completely anonymous; therefore, it did not associate their name from the consent form with responses, nor did it ask for the participant's age. Instead, it asked for their age group, their biological sex, and the evaluation percentage score they received after using PAWDS as the main form of individual identification. The remaining questions asked the participant to rate the following statements on a scale from 1 to 4, with 1 being "Strongly disagree" and 4 being "Strongly agree": "You believe this program can accurately predict whether or not someone has Parkinson's disease."; "You believe doctors and other health care officials would benefit from having this program."; and "You believe this program was difficult to use."

The final two statements given were based on the evaluation percentage score the participant entered, yet they both essentially ask the same question. If their score was below 60 percent, they were prompted to rate the statement, "If you had scored higher on this test, you would consider going to the doctor's to get evaluated for Parkinson's disease." However, if they scored 60 percent or above, they were asked to rate the statement, "After taking the test, you would now consider going to the doctor's to get evaluated for Parkinson's disease." In spite of their relative sameness, these statements were broken up so as to prevent the score the user received being the determining factor for whether or not they feel inclined to seek a formal evaluation for PD. Rather, it begged the user to hypothetically analyze if this is the kind of decision they would make after using the program, untethered from the actual score they obtained. This was extremely crucial to consider, as these statements were foundational for answering the research inquiry.

\section{Results and Analysis/Discussion}

The following section consists of graphs that convey how the participants of this study rated the statements outlined in the Google Forms survey. They are stratified between the three outlined age groups so as to enhance readability and to more easily identify patterns and trends. In this study, I garnered feedback from 30 participants (ten from each age group), 70 percent of which were male and 30 percent female. 


\section{Statement \#1/\#2}

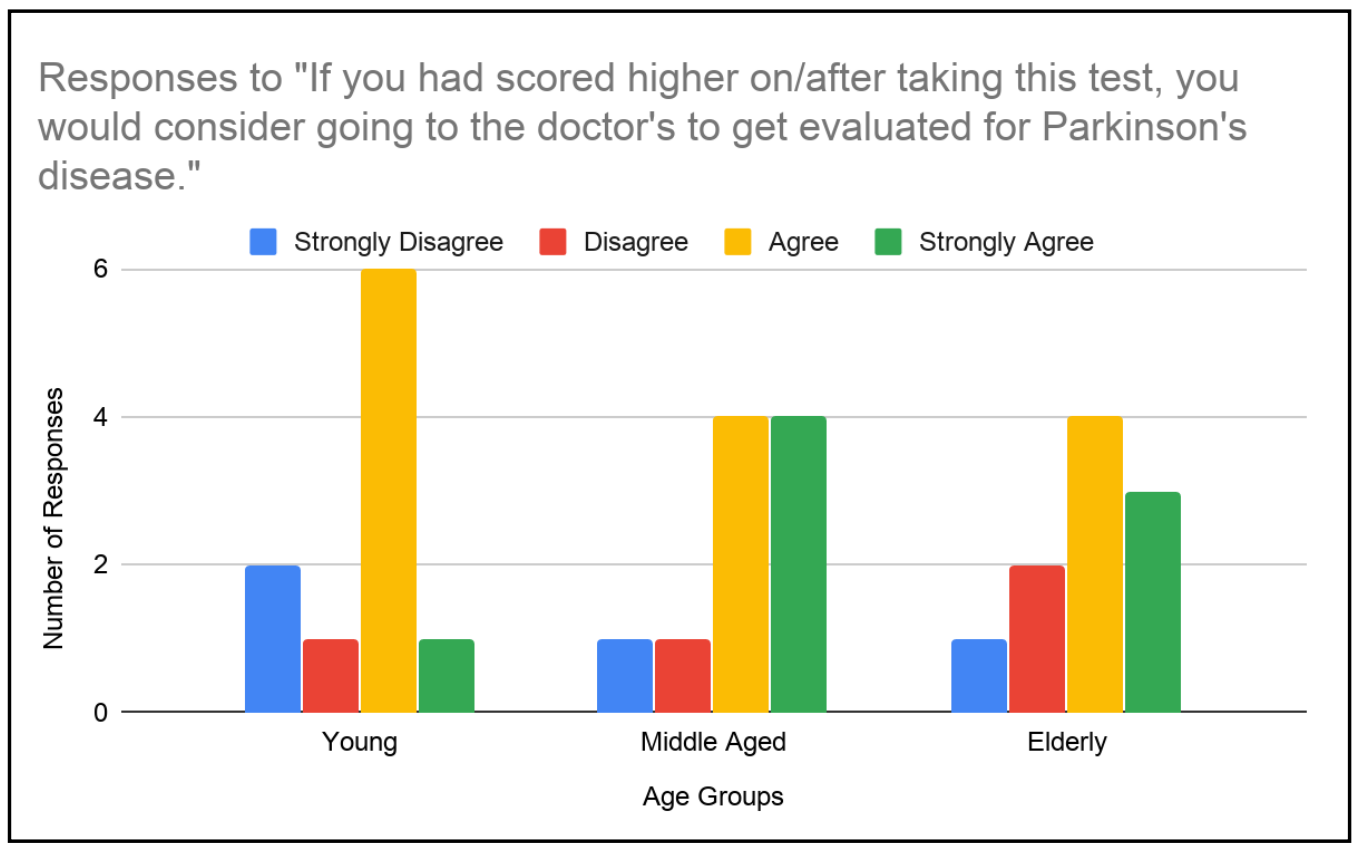

Out of all the statements presented in the survey, these ones were the most pivotal because they hold the conclusion to my research inquiry: to what extent can an algorithmic program that uses handwriting alone estimate the likelihood of a user having PD encourage them to seek a formal diagnosis for the disease? What the responses from the sample participants reveal is that there is a significant trend of agreeing to strongly agree with the statements, as the majority of the responses follow distributions that are skewed to the left.

However, one noteworthy detail can be examined in the "Young" age group, where more participants strongly disagreed with the statement than anyone else in any other group. While this is to be partially expected of this group since this study had more of an elderly focus, it presents a remarkable danger to the future of this type of software. If children and early adults do not feel compelled by this program now, there is a fair probability that they will not come to be influenced by it in the future, which would only help to increase the number of individuals who go undiagnosed with PD in the future. Therefore, one possible way to decrease the number of young people strongly disagreeing with the statements is by educating them beforehand on the nature of software, such as how it is programmed, what studies it is based off of, and how reliable it is. None of this information was revealed to the participants beforehand in order to eliminate any additional bias in the research collection process, but perhaps in a future study it would be wise to educate the participants extensively beforehand about the program and its influences in order to compare it with this study and see if more people, not just young people, end up agreeing to strongly agreeing with the survey statements. 


\section{Statement \#3}

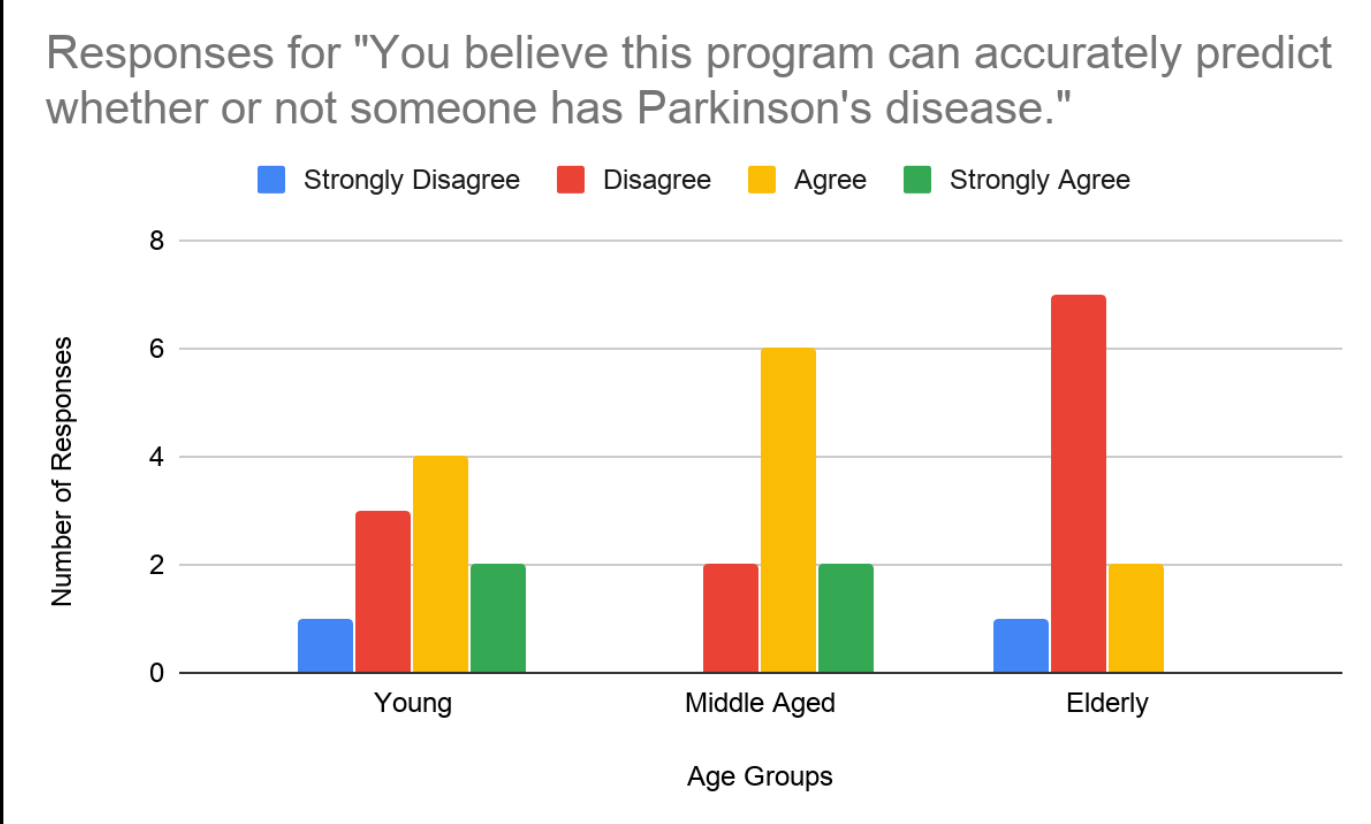

The original motivation behind including this statement was that it would provide further interpretation of the results through correlation with the previous statements. However, the painted picture here drastically differs from what was anticipated. For instance, the distributions for each age group appear to be more Normally distributed and unimodal compared to the more skewed nature of the previous graph. Additionally, the average ratings for the Young, Middle Aged, and Elderly age groups were 2.7, 3.0, and 2.1, respectively. Due to these low ratings, it can be argued that there exists a slight negative correlation between considering going to the doctor's to get evaluated for PD after using PAWDS and actually believing the program can accurately predict whether or not someone has PD.

What this likely means is that there was a disconnect between the user and the software, likely due to either the quality of the program or its general ease of use. As was mentioned in the "Methods" section, PAWDS was developed by myself, who is not a professional when it comes to programs of this caliber, but since this kind of technology is neither ubiquitous nor readily available, I was left with no other option. As such, certain sacrifices had to be made, whether it was with the design of the user interface or how the program worked. For this reason, some users might have felt that the software's amateurish quality was indicative of unreliability or false evaluations. Furthermore, many users likely found the digitizing drawing tablet to be far less intuitive than simply using a touchscreen tablet or a pencil and paper, thus they might have been led to believe that their results were not true to their actual handwriting.

In order to improve this aspect of the research and the software's ability to motivate people to seek a formal diagnosis for PD, it is important to experiment in future studies with programs that have a more glamorous or official user interface so as to convince the users that it is reliable. Additionally, perhaps future iterations of the software can be created without a drawing tablet and instead with a touchscreen tablet so the users can draw directly onto the test screens, thereby making the process all the more intuitive and reputable. Such changes to the software can be made by outsourcing the development of it to a third party institution or by simply upgrading the code base I originally developed. By incorporating these changes, it is likely that more users of all age groups will find the program to be professional and capable of providing an accurate evaluation. 


\section{Statement \#4}

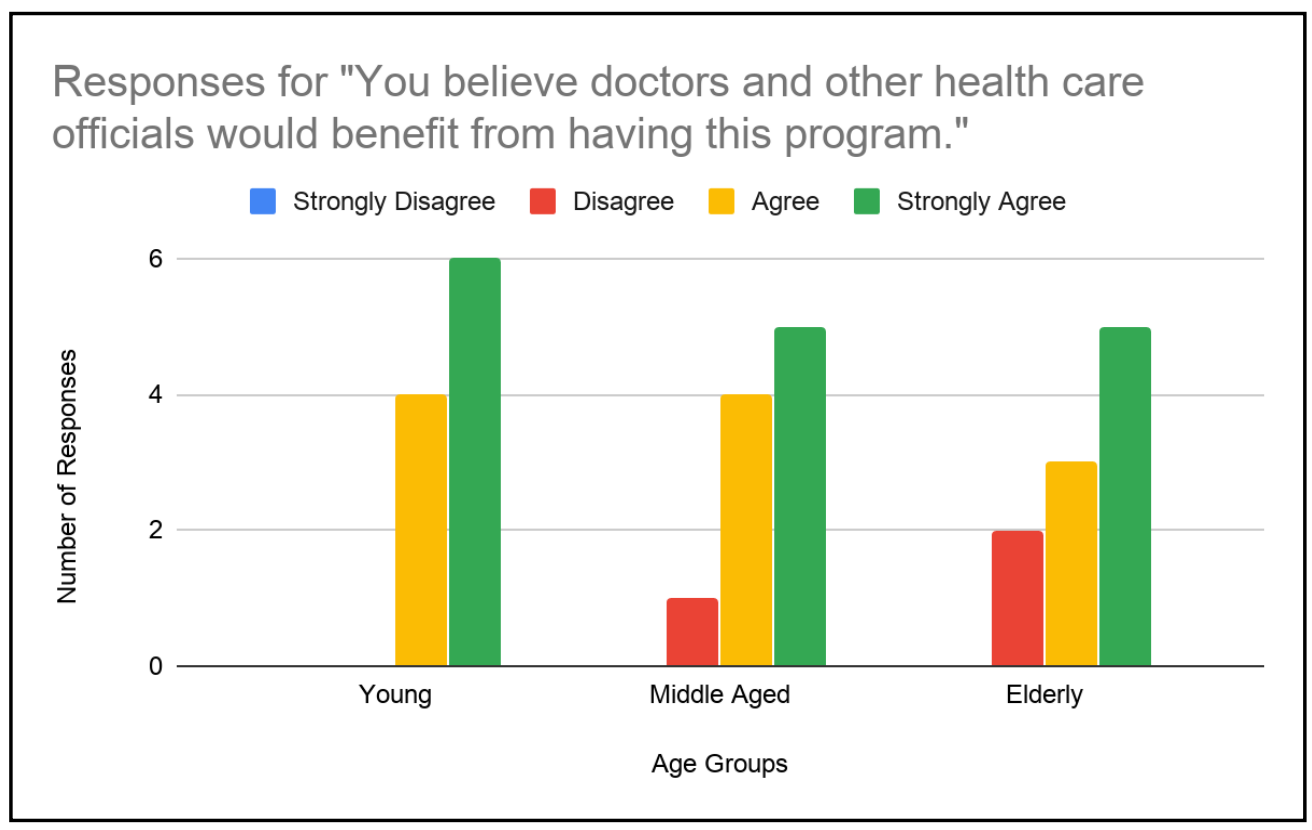

This statement chiefly analyzes the extent to which the participants recognized value in the program for not just the medical world, but also as a whole. The importance of doctors, neurologists, and health care specialists appreciating and utilizing a PD detection program cannot be understated, for its success in the world as a whole is positively correlated with how much the medical sphere finds value in it. For example, a doctor who incorporates it into their diagnostic process could ask their patients to take it in either their waiting rooms or during the actual evaluation itself. Not to mention that if medical professionals supported and encouraged the use of the evaluation software, it is fair to assume that more people would feel compelled to take it since it is being recommended by professionals in the field. As a result, this would possibly lead to an increase in the number of people seeking a formal evaluation and diagnosis for PD.

What is additionally notable in the results of this survey question is the fact that the young age group only agreed with the statement, not disagreeing at all. This observation could be indicative of younger generations identifying the potential of emerging technologies more rapidly than someone older who is not as exposed to tech on a daily basis as young people are. Such a notion is advantageous to the growth of this software, too, because it might motivate youth to invest in its research more willingly, and as they age upwards, they might be more inclined to utilize it themselves. 


\section{Statement \#5}

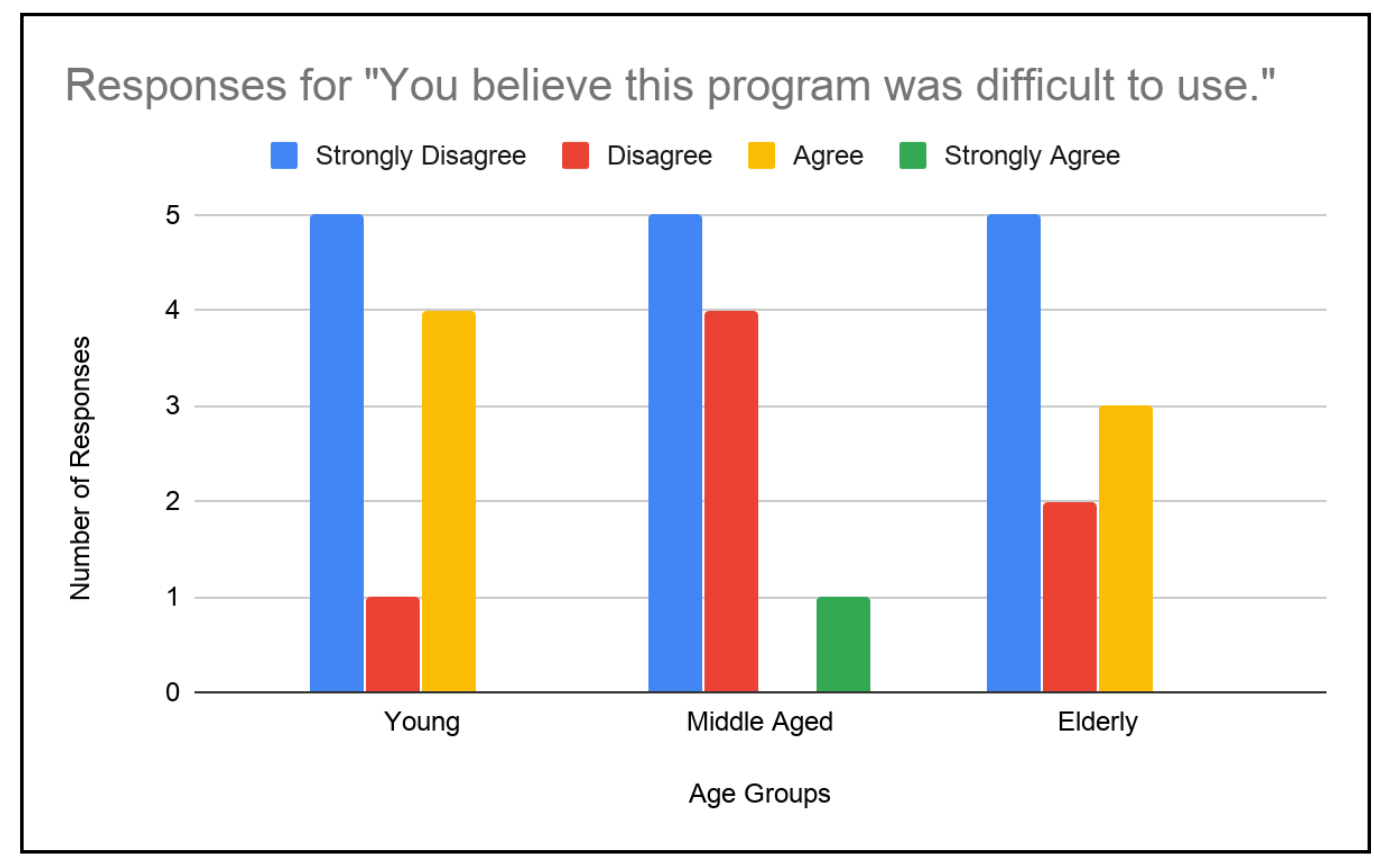

This statement also assisted in the evaluation of participant satisfaction with their use of the program, and it was meant to help construct a correlation between considering going to the doctor's to get evaluated for PD after using PAWDS and how difficult the user thought the program was to operate. However, the data collected happened to be immensely varied, as it is difficult to discern a unique trend anywhere in the graph without the presence of any skewness. The only characteristic of substantial note is the profound quantity of individuals in all age groups who strongly disagreed with the statement; more people strongly disagreed with the idea that the program was difficult to use than any other rating presented. This trend is quite similar to the trend seen in Statement \#3, and it is assumed that the variability and the "Strongly Disagree" votes seen there were caused in large part by how difficult the program was perceived to use. Again, this dissatisfaction with the program can be primarily associated with the user interface and the fact that the program utilized a drawing tablet, which can be unconventional for most users. Therefore, it is important that these aspects are improved upon in future iterations of the program and research study.

However, another area of contention that produced these results might have, again, been the lack of education provided beforehand on the nature of the software and how it works. While I did provide some general instructions on how to use the program, perhaps what I said was inefficient and led to more confusion than what was necessary. In that case, future prototypes should also include a few instructional screens before the test starts that clearly elaborate how the program works, what it is for, and how to perform each of the handwriting tests it provides. With that in mind, additional accessibility features should ultimately be added for people who are hard of hearing, have poor eyesight, or are even color blind. These extra features will ensure, especially with the elderly population, that everyone can use the evaluation software without any unnecessary hindrances or complications.

\section{Conclusion}

PD is an immensely complex disease that manifests itself in a variety of ways, primarily through motor debilitation. By virtue of this notion, the medical world, since the earliest writings of James Parkinson himself, has identified the presence of bradykinesia, resting tremor, and rigidity as the quintessence of formal clinical PD diagnosis. However, with recent innovations in computational algorithmic techniques and new research confirming the correlation between 
micrographia and parkinsonism, handwriting has emerged as the foremost measure of PD. Regardless, a pressing phenomenon still wages on: the prevalence of individuals who go undiagnosed with PD. Therefore, given current technological advancements, an algorithmic software program that predicts a person's likelihood of having PD through their handwriting alone can offer solace in the face of this crippling issue. Since it only assesses the quantifiable kinematics of handwriting, the diagnosis of individuals is made more concrete and definitive, which cannot be said of other diagnostic methods. Additionally, the inherent simplicity and inexpensive nature of this program will attract individuals that are deterred by the currently prodigious cost of healthcare in America.

Through the methods established in this research, the PAWDS prototype that was used, and the data collected therefrom, it can be ultimately concluded that an algorithmic program that uses handwriting alone to evaluate the likelihood of a user having PD has the potential to encourage them to seek a formal diagnosis for the disease, however in its present state its influence is limited.

As it has been stated previously, the amateur tinge of PAWDS still leaves much to be desired. For instance, to simply ignore the quantity of participants who believed the program was, in fact, difficult to use would be a glaring oversight in this report. Indeed, only eight participants agreed with Statement \#5, but their difficulties highlighted a prominent issue with the entire program that will need to be avoided in future prototypes and research studies. Unsurprisingly, people who are unaccustomed to technology have an increasingly challenging time learning how to operate a digitizing drawing tablet. So requiring this tool to be used in PAWDS actually hindered some participants from completing the handwriting tests as efficiently as they desired, which consequently led to some less than accurate evaluation scores. Due to this issue, it would be optimal to discard the drawing tablet and PC software application and replace it with a mobile touch screen app. This feature of being able to draw directly on the drawing space as opposed to approximating the location of the cursor in relation to the position of the stylus on the drawing tablet would not only increase the accuracy of the program's evaluation but would also heighten user satisfaction in the process; hence it should be considered for future prototypes and research studies.

Additionally, it is worth noting that the handwriting tests themselves elicited some frustrations in the users. Due to the way it was programmed, PAWDS will prompt an error if the user lifts up their pen on a test when they were not supposed to (e.g., the circle test). Since technology is prone to failure, unintentional, incorrect drawing inputs caused by irregularities in the drawing tablet can produce erroneous evaluation scores and dissatisfied users. Fortunately, the transfer to a touch screen app, as mentioned previously, would help ease these frustrations, as devices such as iPads are far more accurate with stylus inputs than the drawing tablet that was used in this study. Thus, this should be the standard for all PD detection software and associated research moving forward. Also, the results of these methods were meant to be generalized to the entirety of the American population, young to elderly, yet the limited number of responses collected during this research sequence would suggest a generalization to only a single city's population. This conclusion is not satisfactory enough for the purpose of this research inquiry because the sheer quantity of undiagnosed people, as mentioned in the Literature Review, is rampant among the entirety of the United States population, not just Nevada. Therefore, in future research studies, it is important to have an increased sample population of around 1,000 randomly selected individuals in a multitude of states nationwide, which would provide a data set that could be more faithfully generalized to the country.

Nevertheless, the data collected suggests that making the quality of the program better, making it easier for the general public to use, and having the medical community at large accept it, it is highly possible that this software and many others like it will help increase the number of people who get evaluated and diagnosed for PD. With this conclusion being determined, the literature in this field now has more firm support. For instance, researchers such as Walker et al. and Smits et al. can develop new handwriting detection methods with confidence knowing that their work is receptive to the public. However, it is also now clear that researchers in the field should attempt to avoid digitizing drawing tablets in future detection software, which could have the potential to uproot a multitude of past research due to its prevalent use in the field.

While this program does indeed have remarkable potential, it has yet to be innovated to the point of perfection and dispersed around the world. It takes activism on both sides, those afflicted with PD and those not, young and 
elderly, to promote the mass establishment of this program in easily accessible places so as to increase the number of people who use it and become formally diagnosed with PD. Without this handwriting detection software, a bleak future riddled with the prematurely deceased and the contemporarily tormented on account of undiagnosed PD is woefully inevitable. Fortunately, the answer has been found, and it lies literally within our own hands.

\section{Acknowledgments}

I would like to thank my AP Research instructor Dr. Park for her guidance and encouragement throughout this process. She has witnessed my progression as a writer throughout my high school career, and every step of the way she has been there to praise my efforts. Without her, this paper would still just be a thought waiting to be explored.

Additionally, I would like to send my love and appreciation to my late grandmother Linda King, who inspired me to delve into this research topic in the first place. She grappled with Parkinson's disease throughout the later years of her life, and I witnessed firsthand how it consumed both her body and mind. Unfortunately, she passed away before I could show her the handwriting detection software I programmed and the research I gathered. While her death weighed heavily on me, it gave me a reason to finish this paper. It is my hope that through my research we can eventually reach a day in which no one will ever have to experience what she experienced again.

I love you, grandma.

\section{References}

Berardelli, A., Rothwell, J. C., Thompson, P. D., \& Hallett, M. (2001). Pathophysiology of bradykinesia in Parkinson's disease. Brain, 124(11), 2131-2146.

Berardelli, A., Sabra, A. F., \& Hallett, M. (1983). Physiological mechanisms of rigidity in Parkinson's disease. Journal of Neurology, Neurosurgery \& Psychiatry, 46(1), 45-53.

Carter, S. M. (2019, April 3). Over half of Americans delay or don't get health care because they can't afford it-these 3 treatments get put off most. CNBC. Retrieved from https://www.cnbc.com/2018/11/29/overhalf-of-americans-delay-health-care-becasue-they-cant-afford-it.html

d'Souza, A. D. (2019, April 2). Functional Magnetic Resonance Imaging (fMRI). Retrieved from https://parkinsonsnewstoday.com/parkinsons-disease-tests-diagnosis/functional-magnetic-resonance-imaging-fmri/

De Lau, L. M., \& Breteler, M. M. (2006). Epidemiology of Parkinson's disease. The Lancet Neurology, 5(6), 525-535.

Dementia. (2019, September 19). World Health Organization. Retrieved from https://www.who.int/news$\underline{\mathrm{room} / \text { fact-sheets/detail/dementia }}$

Depression. (2020, January 30). World Health Organization. Retrieved from https://www.who.int/newsroom/fact-sheets/detail/depression

Health resources - Health spending - OECD Data. (2017). Organization for Economic Co-operation and Development. Retrieved from https://data.oecd.org/healthres/health-spending.htm 
Jankovic, J., Schwartz, K. S., \& Ondo, W. (1999). Re-emergent tremor of Parkinson's disease. Journal of Neurology, Neurosurgery \& Psychiatry, 67(5), 646-650.

Lan, K. C., \& Shih, W. Y. (2014). Early Diagnosis of Parkinson's Disease Using a Smartphone. Procedia Computer Science, 34, 305-312.

Nutt, J. G., \& Wooten, G. F. (2005). Diagnosis and initial management of Parkinson's disease. New England Journal of Medicine, 353(10), 1021-1027.

Parkinson, J. (2002). An essay on the shaking palsy. The Journal of neuropsychiatry and clinical neurosciences, 14(2), 223-236.

Parkinson's disease. (2018, June 30). Mayo Clinic. Retrieved from https://www.mayoclinic.org/diseasesconditions/parkinsons-disease/symptoms-causes/syc-20376055

Pointer events. (2020, January 26). MDM Web Docs. Retrieved from https://developer.mozilla.org/enUS/docs/Web/API/Pointer events

Postuma, R. B., Berg, D., Stern, M., Poewe, W., Olanow, C. W., Oertel, W., ... \& Halliday, G. (2015).

MDS clinical diagnostic criteria for Parkinson's disease. Movement Disorders, 30(12), 1591-1601.

Smits, E. J., Tolonen, A. J., Cluitmans, L., van Gils, M., Conway, B. A., Zietsma, R. C., Leenders, K. L., \& Maurits, N. M. (2014). Standardized handwriting to assess bradykinesia, micrographia and tremor in Parkinson's disease. PloS one, 9(5), e97614.https://doi.org/10.1371/journal.pone.0097614

Thomas, M., Lenka, A., \& Kumar Pal, P. (2017). Handwriting Analysis in Parkinson's Disease: Current Status and Future Directions. Movement disorders clinical practice, 4(6), 806-818.

https://doi.org/10.1002/mdc3.12552

Toraman, S., Girgin, M., ÜSTÜNDAĞ, B., \& TÜRKOĞLU, İ. (2019). Classification of the likelihood of colon cancer with machine learning techniques using FTIR signals obtained from plasma. Turkish Journal of Electrical Engineering \& Computer Sciences, 27(3), 1765-1779.

Walker, R. W., Zietsma, R., \& Gray, W. K. (2014). Could a new sensory pen assist in the early diagnosis of Parkinson's?. Expert Review of Medical Devices, 11:3, 243-245, DOI: 10.1586/17434440.2014.900437 